\title{
The boundary condition for vertical velocity and its interdependence with surface gas exchange
}

\author{
Andrew S. Kowalski ${ }^{1,2}$ \\ ${ }^{1}$ Departmento de Física Aplicada, Universidad de Granada, Granada, 18071, Spain \\ ${ }^{2}$ Instituto Interuniversitario de Investigación del Sistema Tierra en Andalucía, Centro Andaluz de Medio Ambiente \\ (IISTA-CEAMA), Granada, 18071, Spain
}

Correspondence to: Andrew S. Kowalski (andyk@ugr.es)

Received: 25 February 2017 - Discussion started: 23 March 2017

Revised: 24 May 2017 - Accepted: 29 May 2017 - Published: 5 July 2017

\begin{abstract}
The law of conservation of linear momentum is applied to surface gas exchanges, employing scale analysis to diagnose the vertical velocity $(w)$ in the boundary layer. Net upward momentum in the surface layer is forced by evaporation $(E)$ and defines non-zero vertical motion, with a magnitude defined by the ratio of $E$ to the air density, as $w=\frac{E}{\rho}$. This is true even right down at the surface where the boundary condition is $\left.w\right|_{0}=\frac{E}{\left.\rho\right|_{0}}$ (where $\left.w\right|_{0}$ and $\left.\rho\right|_{0}$ represent the vertical velocity and density of air at the surface). This Stefan flow velocity implies upward transport of a non-diffusive nature that is a general feature of the troposphere but is of particular importance at the surface, where it assists molecular diffusion with upward gas migration (of $\mathrm{H}_{2} \mathrm{O}$, for example) but opposes that of downward-diffusing species like $\mathrm{CO}_{2}$ during daytime. The definition of flux-gradient relationships (eddy diffusivities) requires rectification to exclude non-diffusive transport, which does not depend on scalar gradients. At the microscopic scale, the role of non-diffusive transport in the process of evaporation from inside a narrow tube - with vapour transport into an overlying, horizontal airstream - was described long ago in classical mechanics and is routinely accounted for by chemical engineers, but has been neglected by scientists studying stomatal conductance. Correctly accounting for non-diffusive transport through stomata, which can appreciably reduce net $\mathrm{CO}_{2}$ transport and marginally boost that of water vapour, should improve characterisations of ecosystem and plant functioning.
\end{abstract}

\section{Introduction}

The vertical velocity $(w)$ is a key variable in the atmospheric sciences, and its precise diagnosis is essential for numerous applications in meteorology. Above the boundary layer, the weather is largely determined by adiabatic adjustments to vertical motion that is slight compared to horizontal winds. Closer to the surface, even a tiny $w$ can result in relevant transport; for example, in a typical boundary layer - with representative temperature $(T=298 \mathrm{~K})$, pressure $(p=101325 \mathrm{~Pa})$ and $\mathrm{CO}_{2}$ mass fraction $\left(607 \mathrm{mg} \mathrm{kg}^{-1}\right.$; a molar ratio of about $400 \mathrm{ppm})-$ just $61 \mu \mathrm{m} \mathrm{s}^{-1}$ of average vertical velocity is needed to waft a biologically significant $44 \mu \mathrm{gCO}_{2} \mathrm{~m}^{-2} \mathrm{~s}^{-1}$ (a $\mathrm{CO}_{2}$ molar flux density of $1 \mu \mathrm{mol} \mathrm{m}{ }^{-2} \mathrm{~s}^{-1}$ ). Modern anemometry cannot resolve such a minuscule airflow (Lee, 1998), and generally $w$ is immensurable at many scales so that it must be derived from other variables (Holton, 1992). Such diagnostic estimation is traditional in synoptic meteorology, but has been developed less rigorously near the surface boundary.

The characterisation of boundary conditions for state and flow variables, in order to enable atmospheric modelling at larger scales, is a fundamental goal of micrometeorology. Since $w$ is air velocity, its boundary condition $\left.w\right|_{0}$ describes the surface-normal or vertical motion of the gas molecules found closest to the surface (at some height $\left.z\right|_{0}$, very nearly but not exactly zero). The Navier-Stokes equations, when applied to the lower atmosphere, are particularly sensitive to the conditions specified at the boundary (Katul et al., 2004), and this lends great importance to $\left.w\right|_{0}$ in the context of dynamic 
modelling. Nevertheless, until now $\left.w\right|_{0}$ has received inadequate attention in boundary-layer meteorology.

Micrometeorologists have made presuppositions regarding $\left.w\right|_{0}$ without formal justification and in contradiction to deductions from classical mechanics. The traditional hypothesis about near-surface winds is that they flow parallel to underlying terrain (Kaimal and Finnigan, 1994; Wilczak et al., 2001) and vanish at the surface (Arya, 1988), implying $\left.w\right|_{0}=0$. This assumption underlies many derivations and abets the prevailing belief that vertical exchanges are accomplished purely by molecular diffusion within a millimetre of the surface (Foken, 2008) or purely by turbulent diffusion at heights of metres or more within the atmospheric boundary layer. However, such a premise is inconsistent with net surface gas exchange (predominantly evaporative), which implies Stefan flow with a mean velocity component normal to the surface. Net mass transfer across a surface results in a velocity component normal to the surface, and an associated non-diffusive flux in the direction of mass transfer (Kreith et al., 1999). The existence and relevance of Stefan flow - first derived and described in the 19th century is certain. Indeed, engineers necessarily account for its role in heat and mass transfer (Abramzon and Sirignano, 1989) when precisely controlling industrial processes that include phase change, such as combustion. For these reasons, it is to be expected that a more accurate means of estimating $\left.w\right|_{0}$ for the atmospheric boundary layer can be achieved by rigorous examination of known surface flux densities in the light of physical laws.

The remaining sections of this work aim to diagnose a defensible lower boundary condition for the vertical velocity $\left(\left.w\right|_{0}\right)$ and to interpret its significance. Section 2 presents the theory and illustrates types of mass transport and heat exchange in fluids via an example from the liquid phase. In Sect. 3, an analytical framework is established and conservation of linear momentum is applied to derive $\left.w\right|_{0}$ from published magnitudes of surface gas exchanges, demonstrating that it is directly proportional to the evaporative flux density $(E)$, consistent with the findings of Stefan. The derived vertical velocity is seen to be relevant in defining the mechanisms of gas transport, which is not accomplished by diffusion alone - even at the surface interface. Section 4 highlights the need to rectify flux-gradient relationships by taking into account the non-diffusive component of transport; this includes boundary-layer similarity theory and physiological descriptions of stomatal conductance. Thus, the implications of these analyses are broad and interdisciplinary.

\section{Theory}

The objective of this section is to establish the theoretical bases for the analyses and interpretations that follow. It opens with a list of symbols (Table 1) along with the meaning and SI units of each variable represented, and finishes with a sum- mary of the most salient points regarding physical laws and transport mechanisms to be recalled in Sect. 3 .

\subsection{Relevant scientific laws}

\subsubsection{The law of conservation of linear momentum}

The principle of conservation of momentum is most fundamental in physics, more so than even Newton's first law (Giancoli, 1984). It defines the momentum of a system of particles as the sum of the momenta of the individual components and establishes that this quantity is conserved in the absence of a net external force. Accordingly, in atmospheric dynamics (Finnigan, 2009) a system may be defined as the $N$ component gas species comprising a particular mass of air, with a net vertical flux density of

$w \rho=\sum_{i=1}^{N} w_{i} \rho_{i}$.

In Eq. (1), $w$ and $\rho$ represent the vertical velocity and density of air, respectively, while $w_{i}$ and $\rho_{i}$ are the properties of component $i$, the species flux density of which is $w_{i} \rho_{i}$. For this species $i$, total transport $w_{i} \rho_{i}$ can be attributed to mechanisms that are diffusive (if $w_{i} \neq w$ ), non-diffusive (if $w \neq 0$ ) or more generally a combination of these two types of transport. Dividing Eq. (1) by the net air density defines the system's vertical velocity as a weighted average of its components (Kowalski, 2012), where the weighting factors are the species' densities.

\subsubsection{The zeroth law of thermodynamics}

The zeroth law establishes the temperature as the variable whose differences determine the possibility for heat exchange between thermodynamic systems. For two systems in thermal contact, if they have the same temperature then they are in thermodynamic equilibrium and therefore exchange no heat. If their temperatures differ, then heat will be transferred from the system with the higher temperature to that with the lower temperature. Heat transfer by molecular conduction depends on gradients in the temperature; in compressible fluids like air, however, turbulent diffusion can occur without thermal contact and yet bring about heat transfer as determined by gradients in the potential temperature (Kowalski and Argüeso, 2011), accounting for any work done/received during the expansion/compression associated with vertical motions.

\subsubsection{Fick's first law of diffusion}

Molecular diffusion has no effect on the net fluid momentum, but "randomly" redistributes fluid components and can cause different species to migrate in different directions, according to component scalar gradients. Regrettably, scientific literature contains inconsistencies regarding the scalar gradient that determines diffusion in the gas phase (Kowalski and 
Table 1. List of symbols, with their meanings and units.

\begin{tabular}{|c|c|c|c|}
\hline Symbol & Variable represented & SI units & Tensor order \\
\hline \multicolumn{4}{|c|}{ General variable representations } \\
\hline$\xi$ & An arbitrary magnitude (can represent any scalar variable) & Depends on $\xi$ & 0 (scalar) \\
\hline$\xi_{i}$ & The magnitude of arbitrary variable $\xi$ for gas species $i$ & Depends on $\xi$ & 0 (scalar) \\
\hline$\nabla \xi$ & The spatial gradient in arbitrary variable $\xi$ & Depends on $\xi$ & 1 (vector) \\
\hline$\left.\xi\right|_{0}$ & The lower boundary condition for arbitrary variable $\xi$ & Depends on $\xi$ & 0 (scalar) \\
\hline \multicolumn{4}{|c|}{ Specific variable representations } \\
\hline$\Delta x, \Delta y$ & Horizontal dimensions of an analytical volume & $\mathrm{m}$ & 0 (scalars) \\
\hline$\delta z$ & Vertical dimension (thickness) of an analytical volume & $\mathrm{m}$ & 0 (scalar) \\
\hline$E$ & Evaporative flux density across a horizontal surface & $\mathrm{kg} \mathrm{m}^{-2} \mathrm{~s}^{-1}$ & 0 (component) \\
\hline$e_{\mathrm{S}}$ & Saturation vapour pressure & $\mathrm{Pa}$ & 0 (scalar) \\
\hline$f$ & Mass fraction & Non-dimensional & 0 (scalar) \\
\hline$F_{i}$ & Vertical flux density of gas species $i$ & $\mathrm{~kg} \mathrm{~m}^{-2} \mathrm{~s}^{-1}$ & 0 (component) \\
\hline$F_{i, \text { non }}$ & Non-diffusive component of $F_{i}$ & $\mathrm{~kg} \mathrm{~m}^{-2} \mathrm{~s}^{-1}$ & 0 (component) \\
\hline$i$ & Index for counting gas species (as in Table 2) & - & 0 (scalar) \\
\hline$K$ & Molecular diffusivity & $\mathrm{m}^{2} \mathrm{~s}^{-1}$ & 0 (scalar) \\
\hline LAI & Leaf area index & Non-dimensional & 0 (scalar) \\
\hline$p$ & Pressure & $\mathrm{Pa}$ & 0 (scalar) \\
\hline$q$ & Specific humidity & Non-dimensional & 0 (scalar) \\
\hline$\rho$ & Air density & $\mathrm{kg} \mathrm{m}^{-3}$ & 0 (scalar) \\
\hline$\sigma$ & Stomatal fraction of leaf area & Non-dimensional & 0 (scalar) \\
\hline$T$ & Air temperature & $\mathrm{K}$ & 0 (scalar) \\
\hline$t$ & Time & s & 0 (scalar) \\
\hline$t_{0}$ & Initial instant of a case scenario & $\mathrm{s}$ & 0 (scalar) \\
\hline$t_{\mathrm{eq}}$ & Equilibrium instant of a case scenario & $\mathrm{s}$ & 0 (scalar) \\
\hline$t_{\mathrm{f}}$ & Final instant of a case scenario & s & 0 (scalar) \\
\hline$v$ & Air velocity & $\mathrm{ms}^{-1}$ & 1 (vector) \\
\hline$w$ & Vertical component of $v$ & $\mathrm{~ms}^{-1}$ & 0 (component) \\
\hline WUE & Water use efficiency & Non-dimensional & 0 (scalar) \\
\hline$z$ & Height above the surface & $\mathrm{m}$ & 0 (component) \\
\hline
\end{tabular}

Argüeso, 2011). The proper form of Fick's first law for diffusion in the vertical direction is

$F_{i, \mathrm{M}}=-\rho K \frac{\partial f_{i}}{\partial z}$

where $F_{i, \mathrm{M}}$ is the vertical flux density of species $i$ due to molecular diffusion, which is proportional to the vertical gradient in that species' mass fraction ( $f_{i}$; Bird et al., 2002), and $z$ is height. Also relevant are the fluid density $(\rho)$ and molecular diffusivity $(K)$. However, $\rho$ must not be included in the derivative in Eq. (2), unless for the trivial case in which it is constant (in an incompressible fluid); in compressible media, gradients in gas density can arise, with no direct relevance to diffusion, due to gradients in pressure or temperature as described by the ideal gas law. It is relevant to note that Adolf Fick arrived at this law, not by experimentation, but rather by analogy with Fourier's law for heat conduction (Bird et al., 2002). By the same analogy, the product of the diffusivity with the scalar gradient in Eq. (2) yields a kinematic flux, which requires multiplication by the fluid density in order to yield the flux density of interest.
Fluxes due to molecular diffusion are referenced to the motion of the fluid's centre of mass or "mixture velocity" (Bird et al., 2002). The simplest example for describing this is that of binary diffusion, where only two species compose the fluid, as in the traditional meteorological breakdown of air into components known as dry air and water vapour. In the case of static diffusion, the fluid velocity is zero and the mass flux of one gas species (water vapour) counterbalances that of the other (dry air). When diffusion occurs in a dynamic fluid (non-zero velocity), then overall transport must be characterised as the sum of diffusive and non-diffusive components.

Turbulent diffusion is analogous to molecular diffusion in the sense that fluid components are randomly redistributed, with different species migrating as a function of gradients in their mass fractions. The primary difference is that eddies rather than molecular motions are responsible for mixing, and the eddy diffusivity (the value of $K$ in Eq. 2, describing $K$-theory; Stull, 1988) is a property of the flow rather than the fluid. The Reynolds number describes the relative importance of molecular and turbulent diffusion, which are other- 


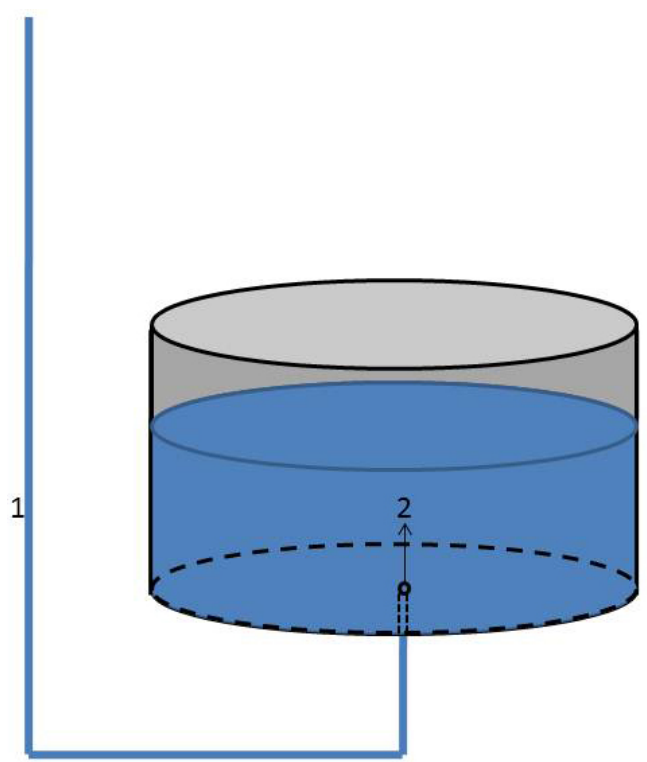

Figure 1. A pool of water being fed from below by a tube. The points indicate water (1) in the tube and (2) in the pool. The arrow indicates the direction of flow.

wise indistinct with respect to the analyses that follow, and will simply be grouped and referred to as "diffusive transport".

\subsection{Transport processes}

In this section, two case studies from the liquid phase will help to identify and define non-diffusive and diffusive types of transport, as well as their scalar source/sink determinants. Let us consider the case of freshwater $\left(35 \times 10^{-5}\right.$ mass fraction of salt) with constant temperature and composition flowing through a tube into the bottom of a pool (Fig. 1) of salinity specified according to the two case scenarios defined below. Considering only flow within the tube (at point 1 ), whether laminar or turbulent, it clearly realises non-diffusive transport of salt, since the salt has no particular behaviour with respect to the fluid, but simply goes with the flow. There are no scalar gradients within the tube, and so there is neither diffusion nor advection. Let us now describe diffusive transport processes within the pool (at point 2), and the nature (whether absolute or relative) of the relevant fluid properties whose gradients determine them by defining sources/sinks, using two illustrative case scenarios.

The temperature is constant in time and space, but other characteristics of the two case scenarios are chosen to elucidate the relationship between diffusive transport processes and scalar gradients:

1. Due to surface evaporation that balances the mass input from the tube, the pool mass is constant. The water remains isothermal by surface heating, which supplies the (latent) energy for evaporation. Initially $\left(t_{0}\right)$ the pool has zero salt mass, but salinity increases constantly, equalling that of the tube water at some moment $\left(t_{\mathrm{eq}}\right)$ and rising by another 2 orders of magnitude to reach that of sea water $\left(35 \times 10^{-3}\right)$ by the end of the scenario $\left(t_{\mathrm{f}}\right)$. This case is of interest from both (a) salt/solute and

(b) thermodynamic points of view:

a. In solute terms, the tube represents a source of (absolute) salt to the pool, but not always of (relative) salinity. Initially $\left(t_{0}\right)$, the water from the tube is more saline than that in the pool, such that nondiffusive and diffusive transport processes operate in tandem to transport salt from the tube upward into the pool; at this moment, the tube is a source of salinity. Salinity advection, defined as the negative of the inner product of two vectors (the velocity with the salinity gradient, with opposite signs), is then positive. Ultimately, however (at $t>t_{\mathrm{eq}}$ ), the water in the pool is more saline than that entering from the tube, such that non-diffusive and diffusive salt transport are in opposite directions. Then the tube dilutes the pool and is a salinity sink, but still a salt source. Salinity advection at $t_{\mathrm{f}}$ is negative. The pool continues to gain salinity after $t_{\mathrm{eq}}$, despite the diluting effects of the tube, due to the concentrating effects of evaporation, which is the ultimate source of salinity. This distinction matters because the gradients that drive advection and diffusion are those in salinity, a relative (not absolute) salt measure. At $t_{\mathrm{f}}$, the diffusive salinity fluxes are oriented against the flow within the pool (downward, and radially inward towards the diluting tube, despite its being a net salt source). By contrast, non-diffusive transport always goes with the flow and accounts for continued upward and outward salt transport, increasing the salt content at the surface.

b. Although thermodynamically trivial - with no heat exchanges whatsoever within the water as determined by the zeroth law - this case nonetheless illustrates the nature of the scalars that determine heat transfer by advection and diffusion (conduction). The heat content of the pool decreases as it becomes more and more saline, due to the inferior heat capacity of saltwater versus freshwater. Similarly, salt diffusion/advection is initially upward/positive but ultimately downward/negative, yet the corresponding implications regarding heat content fluxes say nothing about the transfer of heat. The point here is that the dynamics of the heat content must not be interpreted in terms of heat fluxes, which was done by Finnigan et al. (2003). For this reason, meteorologists correctly define "temperature advection" (Holton, 1992) based on the thermodynamic relevance of gradients in the variable singled out by the zeroth law. 
2. Let us now specify that the water in the pool has the same (freshwater) salinity as that coming from the tube $\left(35 \times 10^{-5}\right)$. If we furthermore remove both surface evaporation and heating from scenario (1), then the temperature remains constant and the salinity corresponds uniformly to that of freshwater, but the pool accumulates mass. In this case, there are convergences in the non-diffusive transports of water, salt and heat content: fluxes into the pool are positive, while fluxes out are null. However, there are no gradients in temperature or salinity, and so there is neither diffusion nor advection in this scenario. The pool does gain volume (depth) but this is only because the fluid under consideration is incompressible. By contrast, for the gas phase, accumulation of absolute quantities - such as air and trace constituent mass and heat content - can occur in a constant volume context (e.g. at a point in an Eulerian fluid specification) due to convergent, non-diffusive transport that defines compression. In the pool, diffusion and advection are clearly null because they are determined by gradients in the relative trace gas amount, the mass fraction, which is a variable of essential utility for the gas phase because it is immune to the effects of compression.

\subsection{An advection-diffusion synopsis}

The analyses that follow rely on the succeeding key points drawn from Sects. 2.1 and 2.2. Advection and diffusion depend on gradients in scalars with relative rather than absolute natures. In incompressible thermodynamics, the relevant gradients are those in the temperature and not the heat content. For trace constituents, the relevant scalar is the mass fraction (e.g. salinity) and not the species density. Advection and diffusion are otherwise physically very distinct. Like non-diffusive transport, diffusion is a vector whose vertical component is of particular interest in the context of surfaceatmosphere exchange. By contrast, advection is a scalar; for some arbitrary quantity $\xi$, it is defined as the negative of the inner product $\boldsymbol{v} \cdot \nabla \xi$, where $\boldsymbol{v}$ is the fluid velocity and $\boldsymbol{\nabla}$ is the gradient operator. Thus advection, unlike diffusion, is not a form of transport, but rather a consequence of differential transport.

The scenarios depicted above correspond to the incompressible case (liquid). When the effects of compressibility are irrelevant, it can be convenient to add the incompressible form of the continuity equation $(\nabla \cdot v=0)$ to advection yielding $-\nabla \cdot \xi \boldsymbol{v}$, the convergence of a kinematic flux. This is called the "flux form" of advection. For a compressible medium such as the atmosphere, however, if $\xi$ is taken to represent some "absolute fluid property such as the (gas) density" (Finnigan et al., 2003), then the transformation of advection into flux form cannot be justified (Kowalski and Arguieso, 2011), since using the incompressible form of the continuity equation leads to unacceptable errors in conservation equations for boundary-layer control volumes (Kowal-
Table 2. Gas components comprising the system to be examined, and their masses.

\begin{tabular}{lll}
\hline$i$ & Gas & Mass $(\mathrm{kg})$ \\
\hline 1 & Nitrogen $\left(\mathrm{N}_{2}\right)$ & $9.14 \times 10^{-16}$ \\
2 & Oxygen $\left(\mathrm{O}_{2}\right)$ & $2.80 \times 10^{-16}$ \\
3 & Argon $(\mathrm{Ar})$ & $1.56 \times 10^{-17}$ \\
4 & Water vapour $\left(\mathrm{H}_{2} \mathrm{O}\right)$ & $1.61 \times 10^{-17}$ \\
5 & Carbon dioxide $\left(\mathrm{CO}_{2}\right)$ & $7.36 \times 10^{-19}$ \\
6 & Methane $\left(\mathrm{CH}_{4}\right)$ & $1.14 \times 10^{-21}$ \\
7 & Nitrous oxide $\left(\mathrm{N}_{2} \mathrm{O}\right)$ & $5.70 \times 10^{-22}$ \\
8 & Ozone $\left(\mathrm{O}_{3}\right)$ & $4.01 \times 10^{-23}$ \\
\hline
\end{tabular}

ski and Serrano-Ortiz, 2007). By contrast, the expression of advection in flux form can be valid if the scalar $\xi$ is carefully chosen for its immunity to the effects of compression, as is the case for the mass fraction. These generalisations regarding the nature of transport by non-diffusive and diffusive mechanisms, and also the nature of advection, will now be applied to the case of vertical transport very near the surface and the mechanisms that participate in surface exchange, after first deriving the boundary condition $\left.w\right|_{0}$.

\section{Analysis}

\subsection{Framework}

The analysis will focus on a system defined as a mixture of gas molecules of different species, and its momentum will be examined. The system's mass is defined (Table 2) by gas components in a ratio that corresponds quite closely to that of the atmosphere (Wallace and Hobbs, 2006) but updated to more closely reflect actual atmospheric composition. At a representative ambient temperature $(T=298 \mathrm{~K})$ and pressure ( $p=101325 \mathrm{~Pa}$ ), the many millions of molecules forming this system occupy a volume of $10^{-15} \mathrm{~m}^{3}$ with $70 \%$ relative humidity. The system geometry will be specified in four different ways, according to the different spatial scales for which $\left.w\right|_{0}$ is to be described:

a. At the synoptic scale, the volume occupied by the system is a lamina of depth $\delta z \sim 10^{-27} \mathrm{~m}$, bounded above and below by constant geopotential surfaces, with horizontal dimensions ( $\Delta x$ and $\Delta y$ ) of the order of $10^{6} \mathrm{~m}$. The fact that $\delta z$ is thinner than the dimension of a molecule does not matter at all when classifying any and all molecules with centres of mass (points, with neither size nor dimension) occupying the lamina as belonging to the volume.

b. At the micrometeorological scale, the volume overlies a flat surface and is shaped as a rectangular lamina of depth $\delta z \sim 10^{-21} \mathrm{~m}$, with horizontal dimensions $(\Delta x$ and $\Delta y$ ) of $10^{3} \mathrm{~m}$. 
c. At the leaf scale, the volume is a rectangular lamina of depth $\delta z \sim 10^{-11} \mathrm{~m}$, with horizontal dimensions $(\Delta x$ and $\Delta y$ ) of $10^{-2} \mathrm{~m}$.

d. At the microscopic scale of plant stomata, the volume is a cube with $\Delta x=\Delta y=\delta z=10^{-5} \mathrm{~m}$. For the purpose of transitioning between the leaf and microscopic scales, plant pores are assumed to occupy a stomatal fraction $\sigma$ of the leaf surface and yet accomplish all gas exchange. The remaining fraction $(1-\sigma)$ is occupied by a cuticular surface and its gas exchange is assumed to be null (Jones, 1983).

Independent of scale, the base height $\left.z\right|_{0}$ of the volume is the lowest for which only air - and neither ocean wave nor land surface element - occupies the volume. The land/ocean/leaf surface will be assumed to be static (i.e. its vertical velocity is zero), impenetrable to the wind (explicitly neglecting ventilation of air-filled pore space), smooth, level and uniform, all for the sake of simplicity. The temporal framework for the analysis is instantaneous, with no need to choose between Eulerian and Lagrangian fluid specifications.

The direction of momentum transport to be examined is vertical, meaning perpendicular to constant geopotential surfaces and therefore to the underlying surface. At the stomatal scale, the stoma to be examined is situated on the upper side of a flat, horizontal leaf; water vapour exiting the stomatal aperture during transpiration therefore has a positive vertical velocity. These analyses can be generalized to sloping surfaces and/or stomata on the underside of leaves, simply by referring to the surface-normal rather than vertical velocity. Hereinafter, however, the term "vertical" will be employed for conciseness.

\subsection{The vertical velocity at the surface boundary}

Knowledge regarding surface exchange (gas flux densities) has advanced to the point where the boundary condition for the vertical velocity $\left(\left.w\right|_{0}\right)$ can be estimated from conservation of linear momentum - applying Eq. (1) to the system defined in Table 2 - and vastly simplified to a simple function of the evaporation rate $(E)$. The species flux densities $\left(w_{i} \rho_{i}\right)$ within the system represent the surface exchanges of the corresponding gas species $(i)$. Scale analysis of surface gas exchange magnitudes, published from investigations at a particularly well-equipped forest site in Finland (Table 3), reveals that for the water vapour species $(i=4)$, the flux density $\left(E=w_{4} \rho_{4}\right)$ is several orders of magnitude larger than both the flux density of any dry air component species and even the net flux density of dry air. Such dominance by water vapour exchanges is representative of most surfaces worldwide. This is especially so because the two largest dry air component fluxes are opposed, with photosynthetic/respiratory $\mathrm{CO}_{2}$ uptake/emission largely offset by $\mathrm{O}_{2}$ emission/uptake $(\mathrm{Gu}, 2013)$. Hence, following tradition in micrometeorology (Webb et al., 1980), surface exchange of dry air can be neglected, allowing the elimination from Eq. (1) of all species flux densities except for that of water vapour $\left(\mathrm{H}_{2} \mathrm{O} ; i=4\right)$. Therefore, net air transfer across the surface can be approximated very accurately as

$\left.\left.w\right|_{0} \rho\right|_{0}=\left.\left.w_{4}\right|_{0} \rho_{4}\right|_{0}=E$,

where $\left.w_{4}\right|_{0}$ and $\left.\rho_{4}\right|_{0}$ are the $\mathrm{H}_{2} \mathrm{O}$ species velocity and density at the surface. Equation (3) states that, at the surface, the net vertical flux density of air is equal to the net vertical flux density of water vapour, which is the evaporation rate. Solving this for $\left.w\right|_{0}$ allows estimation of the lower boundary condition for the vertical velocity as

$\left.w\right|_{0}=\frac{E}{\left.\rho\right|_{0}}$.

The representative evaporation rate prescribed in Table 3 and vertical velocity resulting from Eq. (4) are valid for most of the scales defined above. Thus, the boundary condition $\left.w\right|_{0}$ is valid for the synoptic scale (notwithstanding vertical motion aloft, such as subsidence), for the micrometeorological scale and even for the leaf scale. In the context of scale analysis, leaves may be approximated as having equal area as the underlying surface (i.e. a unit leaf area index or $\mathrm{LAI}=1$ ), and equal evaporation rates as the surface in general. This latter assumption does not neglect soil evaporation, but only excludes the possibility that it would dominate leaf evaporation by 1 order of magnitude. Thus, it will be assumed here that the assumed evaporation rate and derived vertical velocities are equally valid at synoptic (a), micrometeorological (b) and leaf (c) scales. The order of magnitude is different, however, at the microscopic (d) scale. To show this, it will be assumed here that all leaf evaporation (or transpiration) occurs through the small fraction of the leaf that is stomatal $(\sigma)$, such that both the stomatal evaporative flux density and the lower boundary condition for the vertical velocity $\left(\left.w\right|_{0}\right)$ are a factor $1 / \sigma$ greater than those at larger scales. Independent of scale, Eq. (4) states that, for a positive evaporation rate, the boundary condition for the vertical velocity is non-zero and upward.

Given that the surface boundary is static, it may well be asked why there is a non-zero boundary condition for the vertical velocity of air. The answer is that evaporation induces a pressure gradient force that pushes air away from the surface. Evaporation into air increments the water vapour pressure and thereby the total pressure, according to Dalton's law. If evaporation were to proceed until equilibrium is achieved, the pressure added by evaporation would correspond to the saturation vapour pressure $\left(e_{\mathrm{s}} ;\right.$ Fig. 2$)$. Its temperature dependency has been quantified empirically and is described by the Clausius-Clapeyron relation. It is this evaporationinduced pressure gradient force that pushes the manometer in Fig. 2 to its new position, and similarly drives winds away from the surface. 
Table 3. The first six air components by their surface exchange scale magnitude, and the net exchange of air as the sum of these flux densities. Representative surface exchanges are taken from the Finnish boreal forest site (Suni et al., 2003; Aaltonen et al., 2011). The $\mathrm{O}_{2}$ exchange rate assumes $1: 1$ stoichiometry with $\mathrm{CO}_{2}$.

\begin{tabular}{lrrll}
\hline Gas & $\begin{array}{r}\text { Typical mass flux, } F_{i} \\
\left(\mathrm{mg} \mathrm{m}^{-2} \mathrm{~s}^{-1}\right)\end{array}$ & $\begin{array}{r}\text { Corresponding molar flux } \\
\left(\mathrm{mmol} \mathrm{m}^{-2} \mathrm{~s}^{-1}\right)\end{array}$ & Source & \\
\hline $\mathrm{H}_{2} \mathrm{O}$ & 36 & 2 & Suni et al. (2003) & 4 \\
$\mathrm{CO}_{2}$ & -0.088 & -0.002 & Suni et al. (2003) & 5 \\
$\mathrm{O}_{2}$ & 0.064 & 0.002 & Gu (2013) & 2 \\
$\mathrm{CH}_{4}$ & -0.000032 & -0.000002 & Aaltonen et al. (2011) & 6 \\
$\mathrm{O}_{3}$ & 0.0000096 & -0.0000002 & Suni et al. (2003) & 8 \\
$\mathrm{~N}_{2} \mathrm{O}$ & 0.00000088 & 0.00000002 & Aaltonen et al. (2011) & 7 \\
$\mathrm{Air}$ & 35.98 & - & This study & - \\
\hline
\end{tabular}

Although this upward air propulsion occurs at the surface, air velocities are generally upward throughout the boundary layer in a climatological context. Indeed, the dominant role of water vapour in determining the net vertical momentum of air is a general feature of the troposphere. In the context of the hydrological cycle, water vapour is transported from the surface where it has an evaporative source, to further aloft where clouds develop via processes that act as water vapour sinks: condensation and vapour deposition onto ice crystals (or ice nuclei). In terms of total water, upward transport in the gas phase is offset, over the long term, by downward transport in liquid and solid phases (e.g. rain and snow); unlike the water vapour flux, however, precipitation does not directly define air motion. It is true that downward water vapour transport occurs during dewfall - with surface condensation, as described by Eq. (4) with a negative evaporation rate $(E<0)$ - but this plays a minor role in the global water balance. Generally, the relative magnitudes of gas exchanges used for the scale analysis in Table 3 are representative throughout most of the troposphere, with upward water vapour flux densities dominating those of other gases in the vertical direction. In the surface layer, sometimes termed the "constant flux layer" (Dyer and Hicks, 1970), Eq. (4) can be extrapolated away from the surface under steady-state conditions to yield

$w=\frac{E}{\rho}$.

\subsection{Mechanisms of gas transport at the surface}

Non-zero vertical momentum in the lower atmosphere and right at the surface boundary - dominated by the flux density of water vapour and generally upward due to evaporation means that diffusion is not the lone relevant transport mechanism that participates in surface exchange, as has been generally supposed. This is true for all atmospheric constituents, not only for water vapour; over an evaporating surface, any molecule undergoing collisions with its neighbours does not

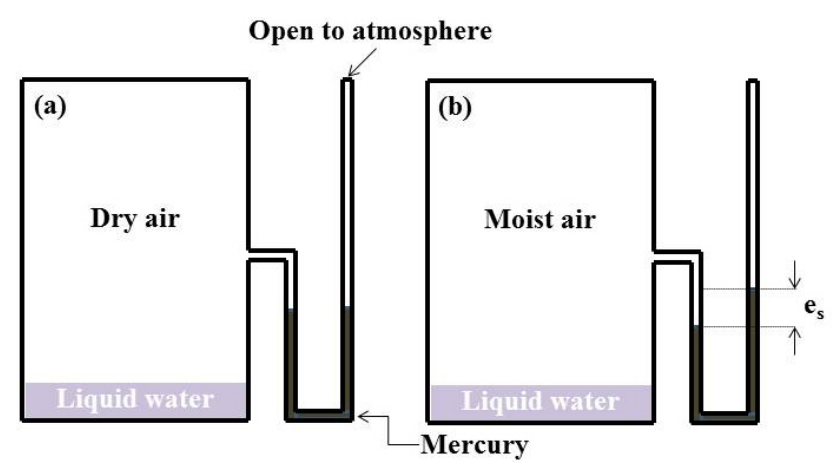

Figure 2. Illustration of evaporation incrementing air pressure. Chamber air evolves from (a) dry air initially at atmospheric pressure to (b) moist air at a pressure that has risen by the partial pressure of water vapour, ultimately at equilibrium (saturation vapour pressure, $e_{\mathrm{S}}$ ). The force generated by evaporation propels the mercury in the manometer from its initial position.

experience a random walk (a characteristic of static diffusion), but rather tends to be swept upward with the flow. The upward air current similarly wafts aerosol particles, although these may move downwards if their fall velocities exceed the upward air motion. The upward flow velocity is rather small - just $31 \mu^{-1}$ for the conditions specified above and the evaporation rate of Table 3, according to Eqs. (4) and (5). It does not exclude the possibility of diffusive transport in any direction, but does imply a relevant, non-diffusive component of transport for any gas, with a magnitude that is not related to the scalar gradient of that gas.

The non-diffusive flux density of species $i$ can be expressed as

$F_{i, \text { non }}=w \rho_{i}$,

and when substituting for $w$ from Eq. (5) this becomes

$F_{i, \text { non }}=E f_{i}$,

that is, the product of the evaporation rate and the species mass fraction. Examination of its magnitude near the surface 
for different gases will now show that, while this is often small in comparison with the diffusive component, it is not negligible in every case, depending on the magnitudes of the mass fraction and surface exchange for the gas considered.

Interpreting decomposed transport is simplest when examining a gas whose surface exchange is very well known, such as the null value for inert Argon (Ar) that constitutes ca. $1.3 \%$ of dry atmospheric mass (Wallace and Hobbs, 2006). Considering the state variables defined by Table 2 and the evaporation rate of Table 3, Eq. (7) indicates $458 \mu \mathrm{g} \mathrm{m}^{-2} \mathrm{~s}^{-1}$ (a molar flux density of $11.6 \mu \mathrm{mol} \mathrm{m}{ }^{-2} \mathrm{~s}^{-1}$ ) of upward, nondiffusive Ar transport $\left(F_{3}\right.$, non $)$. To comprehend this, it helps to recall that the constant addition of $\mathrm{H}_{2} \mathrm{O}$ dilutes dry air at the surface and promotes its downward diffusion. For a null net flux of inert Ar to exist, downward diffusion of this dry air component must exactly cancel the upward non-diffusive transport, and therefore it is $458 \mu \mathrm{g} \mathrm{m}^{-2} \mathrm{~s}^{-1}$ for the state and evaporative conditions specified above. These opposing nondiffusive and diffusive Ar transport processes are quite analogous to case scenario 1 of Sect. 2.2, at the instant $t_{\mathrm{f}}$ when the fluid emitted into the pool has a diluting effect. Such dual transport mechanisms are also relevant for vital gases, with different transport directions and degrees of relevance, depending on the density and flux density of the gas in question.

For $\mathrm{H}_{2} \mathrm{O}$, the two types of gas transport mechanisms operate in tandem, with the non-diffusive component contributing a fraction of upward $\mathrm{H}_{2} \mathrm{O}$ transport that, according to Eq. (7), is exactly the water vapour mass fraction or specific humidity (Wallace and Hobbs, 2006)

$q \equiv f_{4} \equiv \frac{\rho_{4}}{\rho}$.

This is just $2 \%$ for the state conditions previously specified, but can approach $5 \%$ for very warm evaporating surfaces and/or high-altitude environments. The breakdown of $\mathrm{H}_{2} \mathrm{O}$ transport into diffusive and non-diffusive components is analogous to case scenario 1 of Sect. 2.2 at an instant prior to $t_{\mathrm{eq}}$ when the fluid introduced to the pool is highly concentrated in comparison with the fluid already in the pool. In any case, non-diffusive $\mathrm{H}_{2} \mathrm{O}$ transport is generally secondary to diffusive transport, but its neglect in an ecophysiological context can lead to larger relative errors, as will be shown in Sect. 4.

For $\mathrm{CO}_{2}$, which usually migrates downward during evaporative conditions because of photosynthetic uptake, upward transport of a non-diffusive nature is even more relevant, opposing the downward flux due to diffusion. To see this, let us examine the typical gas transport magnitudes of Table 2 and the atmospheric state conditions specified above. According to Eq. (7), non-diffusive $\mathrm{CO}_{2}$ transport $\left(F_{5}\right.$ non $)$ is then $21.5 \mu \mathrm{g} \mathrm{m}^{-2} \mathrm{~s}^{-1}$ (a molar flux density of $0.49 \mu \mathrm{mol} \mathrm{m}^{-2} \mathrm{~s}^{-1}$ ) in the upward direction, requiring that downward $\mathrm{CO}_{2}$ diffusion be $109.5 \mu \mathrm{g} \mathrm{m}^{-2} \mathrm{~s}^{-1}$ in order to yield $88 \mu \mathrm{g} \mathrm{m}^{-2} \mathrm{~s}^{-1}$ of net surface uptake; if not accounting for the non-diffusive resistance to net transport, the $\mathrm{CO}_{2}$ diffusivity would be underestimated by ca. $20 \%$. The case of $\mathrm{CO}_{2}$ uptake is not analogous to any pool/tube scenario in Fig. 1. However, different conditions with equal evaporation $\left(E=36 \mathrm{mg} \mathrm{m}^{-2} \mathrm{~s}^{-1}\right)$ and $\mathrm{CO}_{2}$ emission in the amount of $21.5 \mu \mathrm{g} \mathrm{m}^{-2} \mathrm{~s}^{-1}$ (by respiration, for example) would correspond to the case of zero $\mathrm{CO}_{2}$ diffusion (as at the instant $t_{\mathrm{eq}}$ ), since the $\mathrm{CO}_{2}$ mass fractions of both the atmosphere and the gas mixture emitted by the surface are identical. Viewed in the traditional diffusion-only paradigm, such a situation involving a net flux but no gradient $\left(F_{3}=F_{3}\right.$,non $)$ would require a physically absurd infinite diffusivity. At this same evaporation rate, but with lower $\mathrm{CO}_{2}$ emission, diffusion of $\mathrm{CO}_{2}$ would be downward, towards the surface which is a source of $\mathrm{CO}_{2}$ but a sink of the $\mathrm{CO}_{2}$ mass fraction (analogous to salinity in case scenario 1 of Sect. 2.2 at some instant between $t_{\mathrm{eq}}$ and $t_{\mathrm{f}}$ when the fluid emitted to the pool has a diluting effect). Whatever the direction of net $\mathrm{CO}_{2}$ transport, these case examples demonstrate the need for sometimes substantial rectifications to flux-gradient relationships whether expressed as a conductance, resistance, deposition velocity or eddy diffusivity ( $K$-theory) when correctly accounting for non-diffusive transport.

\section{Discussion}

Relevant transport of a non-diffusive nature implies a need to revise the basis of flux-gradient theory, both in the boundary layer and also at smaller scales regarding gas transfer through plant pores. One of the key goals of micrometeorology has been the derivation of the vertical transports of mass, heat, and momentum from profiles of wind speeds and scalar variables in the boundary layer (Businger et al., 1971). The analyses above elucidate how gradients relate to only the diffusive components of such exchanges. Therefore, non-diffusive flux components must be subtracted out in order to characterise turbulent transport in terms of eddy diffusivities, a key goal of Monin-Obukhov similarity theory (Obukhov, 1971). Perhaps more important is the need to distinguish between non-diffusive and diffusive transport mechanisms prior to assessing molecular diffusivities (conductances).

When Eq. (3) is applied at the stomatal apertures where virtually all plant gas exchanges occur, it is revealed that jets of air escape from these pores during transpiration. In the context of the scale analysis begun in Sect. 3.2, it is appropriate to note that even fully open stomata occupy just $1 \%$ of leaf area (Jones, 1983), leaving $99 \%$ cuticular and inert with regard to vital gas exchanges $(\sigma=0.01)$. As noted in Sect. 3.2, this means that for the microscopic scale (d; Sect. 3.1) of the stomatal aperture, both the local evaporative flux density $(E)$ and therefore the lower boundary condition for the vertical velocity $\left(\left.w\right|_{0}\right)$ predicted by Eq. (4) are 2 orders of magnitude greater than the $31 \mu \mathrm{ms}^{-1}$ estimated above. In other words, a typical average airspeed exiting a stomatal aperture is $3.1 \mathrm{~mm} \mathrm{~s}^{-1}$. For non-turbulent flow 
through a cylindrical tube/aperture (i.e. Poiseuille flow), the velocity at the core of such an air current is twice as large. If a characteristic timescale is defined for air blowing through stomata as the ratio of a typical stomatal aperture diameter (ca. $6 \mu \mathrm{m}$ ) to this core velocity, it is found to be of the order of $10 \mathrm{~ms}$, illustrating that air is expulsed from plants in the form of "stomatal jets". Non-diffusive gas transport by such airflow exiting stomata - assisting with water vapour egress but inhibiting $\mathrm{CO}_{2}$ ingress - has been previously conceived.

The concept of net motion and consequent non-diffusive transport out of stomata is not new, but has been disregarded by plant ecologists. Parkinson and Penman (1970) put forth that the massive water vapour flux from transpiration implies an outbound air current as a background against which diffusion operates. Regrettably, however, their interpretation has largely been forgotten, having been refuted in an analysis (Jarman, 1974) that incorrectly assumed "no net flow of air" - disregarding conservation of momentum - and yet seems to have gained acceptance (von Caemmerer and Farquhar, 1981). Similarly, Leuning (1983) recognised the relevance of non-diffusive transport and furthermore identified excess pressure inside the stomatal cavity as the impetus for the outward airstream (which he termed "viscous flow"), but had little impact on the mainstream characterisation of stomatal conductance. Rather, important aspects of ecophysiology continue to hinge upon the assumption that diffusion alone transports vital gases through plant pores, disregarding both the above-mentioned studies and more importantly the fact that gas transport mechanisms through such apertures were accurately described by one of the great physicists of the 19th century.

Because Josef Stefan substantially helped to establish the fundamentals of classical physics, his name is often mentioned in the same breath as Boltzmann (regarding blackbody radiation) and Maxwell (for diffusion). However, his work in the latter regard has been broadly ignored by scientists studying gas exchanges through plant stomata. Stefan's study of evaporation from the interior of a narrow, vertical cylinder with vapour transport into an overlying, horizontal stream of air is of particular relevance to the discipline of ecophysiology. He determined that this is not a problem of static diffusion, but rather includes an element of non-diffusive transport due to a mean velocity in the direction of the vapour flux, induced by evaporation and now commonly known as Stefan flow. Engineers know this history and refer to such a scenario as a Stefan tube (Lienhard IV and Lienhard V, 2000), and routinely reckon transport by Stefan flow in addition to that caused by diffusion. Such accounting is necessary for precise control in industrial applications such as combustion and is described in many chemical engineering texts (Kreith et al., 1999; Lienhard IV and Lienhard V, 2000; Bird et al., 2002). The phenomenon of transpiration through a stoma is a reasonable proxy for a Stefan tube, the main difference being that evaporation in the Stefan tube depletes the pool of evaporating liquid, whose surface therefore recedes downward. By contrast, the evaporating water in the stomatal cavity is continually replenished by vascular flow from within the plant. If anything, this reinforces the magnitude of the upward vertical air velocity, in contrast to the Stefan tube, and is consistent with that derived from momentum conservation in Eqs. (4) and (5).

Non-diffusive transport by Stefan flow has implications for defining key physiological parameters, which are more significant than the percentages of $\mathrm{CO}_{2}$ and water vapour transport calculated above. Plant physiologists have postulated that stomata act to maximise the ratio of carbon gain to water loss (Cowan and Farquahar, 1977) or water use efficiency (WUE), an ecosystem trait that constrains global biogeochemical cycles (Keenan et al., 2013). In formulating this parameter, presuming molecular diffusion to be the lone transport mechanism, the water vapour conductance is usually taken as 1.6 times that of $\mathrm{CO}_{2}$ (Beer et al., 2009), based on the ratio of their diffusivities - the inverse of the square root of the ratio of their molecular masses, according to Graham's law. Such an assumption underlies the very concept of stomatal control (Jones, 1983), but neglects the role of nondiffusive transport for both gases. Net momentum exiting stomata both expedites water vapour egress and retards $\mathrm{CO}_{2}$ ingress, versus the case of static diffusion, in each case acting to reduce the WUE. Importantly, water vapour transport by stomatal jets depends not only on physiology but also physically on the state variable $q$, according to Eq. (8). Consistent with the determinants of $q$, as the temperature of a (saturated) stomatal environment increases, even for a constant stomatal aperture, the WUE is reduced, wresting some control over gas exchange rates from the plant. Perhaps equally importantly, opposition to $\mathrm{CO}_{2}$ uptake by stomatal jets also should be considered when modelling the most fundamental of biological processes, namely photosynthesis.

Accurate modelling of primary production in plants may require a fuller description of stomatal transport mechanisms, including non-diffusive expulsion by jets. The partial pressure of $\mathrm{CO}_{2}$ inside the stomata is a key input parameter for the classic photosynthesis model (Farquhar et al., 1980), but is never directly measured. Rather, it must be inferred from gas exchange measurements and assumptions about the relative conductance of water vapour and $\mathrm{CO}_{2}$, as described above. The amendment of such calculations to account for non-diffusive transport of both $\mathrm{CO}_{2}$ and $\mathrm{H}_{2} \mathrm{O}$ should help to improve the accuracy of physiological models.

As a final note regarding ecophysiology, studies of plant functioning conducted using alternative gas environments should be interpreted with care. Stomatal responses to humidity variations have been studied in several plant species using the $\mathrm{He}: \mathrm{O}_{2}$ gas mixture termed "helox" (Mott and Parkhurst, 1991). In the context of conservation of linear momentum, it is relevant that the effective molecular weight of helox is just $29 \%$ that of dry air. Under equal conditions of temperature and pressure, helox has far less density, and so during transpiration both $\left.w\right|_{0}$ from Eq. (4) and the non- 
diffusive component of stomatal transport from Eq. (7) are 3.5 times greater than in air. The validity of helox for characterising natural plant functioning is thus dubious due to its low inertia versus that of air.

\section{Conclusions}

Evaporation $(E)$ is the dominant surface gas exchange, and forces net upward momentum in the surface layer such that the lower boundary condition for the vertical velocity is $\left.w\right|_{0}=\frac{E}{\left.\rho\right|_{0}}$, where $\left.\rho\right|_{0}$ is the air density at the surface. This non-zero vertical velocity describes Stefan flow and implies gas exchange of a non-diffusive nature, which must be extracted from the net transport of any gas prior to relating that gas's resultant diffusive transport component to scalar gradients, as in the Monin-Obukhov similarity theory. Such a correction of the flux-gradient theory is of particular importance for descriptions of gas exchange through plant stomata, which should be amended to account for non-diffusive transport by stomatal jets, which help to expel water vapour but hinder the ingress of $\mathrm{CO}_{2}$.

Data availability. No data sets were used in this article.

Competing interests. The author declares that he has no conflict of interest.

Acknowledgements. This work is dedicated, with fondness and great esteem, to the memory of Ray Leuning whose insights led to substantive improvements both in this work and broadly in the science of surface gas exchanges. Investigation into this matter was funded by Spanish national project GEISpain (CGL2014-52838-C2-1-R). The author thanks Penélope SerranoOrtiz, Enrique Pérez Sánchez-Cañete, Óscar Pérez-Priego, Sonia Chamizo, Ana López-Ballesteros, Russell L. Scott, and Jorge Pérez-Quezada and anonymous reviewers for bibliographical guidance, comments and criticisms that helped to clarify the manuscript.

Edited by: Armin Sorooshian

Reviewed by: Werner Eugster and two anonymous referees

\section{References}

Aaltonen, H., Pumpanen, J., Pihlatie, M., Hakola, H., Hellén, H., Kulmala, L., Vesala, T., and Bäck, J.: Boreal pine forest floor biogenic volatile organic compound emissions peak in early summer and autumn, Agr. Forest Meteorol., 151, 682-691, https://doi.org/10.1016/j.agrformet.2010.12.010, 2011.

Abramzon, B. and Sirignano, W. A.: Droplet vaporization model for spray compustion calculations, Int. J. Heat Mass Tran., 32, 1605-1618, 1989.
Arya, S. P.: Introduction to Micrometeorology, Academic Press, San Diego, 307 pp., 1988.

Beer, C., Ciais, P., Reichstein, M., Baldocchi, D., Law, B. E., Papale, D., Soussana, J.-F., Ammann, C., Buchmann, N., Frank, D., Gianelle, D., Janssens, I. A., Knohl, A., Köstner, B., Moors, E., Roupsard, O., Verbeeck, H., Vesala, T., Williams, C. A., and Wohlfahrt, G.: Temporal and among-site variability of inherent water use efficiency at the ecosystem level, Global Biogeochem. Cy., 23, GB2018, https://doi.org/10.1029/2008GB003233, 2009.

Bird, R. B., Stewart, W. E., and Lightfoot, E. N.: Transport Phenomena, John Wiley \& Sons, Cambridge, 2002.

Businger, J. A., Wyngaard, J. C., Izumi, Y., and Bradley, E. F.: Fluxprofile relationships in the atmospheric surface layer, J. Atmos. Sci., 28, 181-189, 1971.

Cowan, I. R. and Farquahar, G. D.: Stomatal function in relation to leaf metabolism and environment, Sym. Soc. Exp. Biol., 31, 471-505, 1977.

Dyer, A. J. and Hicks, B. B.: Flux-gradient relationships in the constant flux layer, Q. J. Roy. Meteor. Soc., 96, 713-721, 1970.

Farquhar, G. D., von Caemmerer, S., and Berry, J. A.: A biochemical model of photosynthetic $\mathrm{CO}_{2}$ assimilation in leaves of $\mathrm{C}_{3}$ species, Planta, 149, 78-90, 1980.

Finnigan, J. J.: Response to comment by Dr. A.S. Kowalski on "The storage term in eddy flux calculations", Agr. Forest Meteorol., 149, 725-729, 2009.

Finnigan, J. J., Clement, R., Malhi, Y., Leuning, R., and Cleugh, H. A.: A re-evaluation of long-term flux measurement techniques. Part I: Averaging and coordinate rotation, Bound.-Lay. Meteorol., 107, 1-48, 2003.

Foken, T.: Micrometeorology, Springer-Verlag, Berlin, 306 pp., 2008.

Giancoli, D. C.: General Physics, Prentice-Hall, Englewood Cliffs, NJ, 1984.

$\mathrm{Gu}$, L.: An eddy covariance theory of using $\mathrm{O}_{2}$ to $\mathrm{CO}_{2}$ exchange ratio to constrain measurements of net ecosystem exchange of any gas species, Agr. Forest Meteorol., 176, 104-110, https://doi.org/10.1016/j.agrformet.2013.03.012, 2013.

Holton, J. R.: An Introduction to Dynamic Meteorology, Academic Press, San Diego, 511 pp., 1992.

Jarman, P. D.: The diffusin of carbon dioxide and water vapour through stomata, J. Exp. Bot., 25, 927-936, 1974.

Jones, H. G.: Plants and microclimate: a quantitative approach to environmental plant physiology, Cambridge University Press, New York, 323 pp., 1983.

Kaimal, J. C. and Finnigan, J. J.: Atmospheric Boundary Layer Flows Their Measurement and Structure, Oxford University Press, New York, 242 pp., 1994.

Katul, G., Cava, D., Poggi, D., Albertson, J., and Mahrt, L.: Stationarity, homogeneity, and ergodicity in canopy turbulence, in: Handbook of Micrometeorology, edited by: Lee, X., Massman, W., and Law, B., Kluwer Academic, New York, 29, 161-180, 2004.

Keenan, T. F., Hollinger, D. Y., Bohrer, G., Dragoni, D., Munger, J. W., Schmid, H. P., and Richardson, A. D.: Increase in forest water-use efficiency as atmospheric carbon dioxide concentrations rise, Nature, 499, 324-327, https://doi.org/10.1038/nature12291, 2013.

Kowalski, A. S.: Exact averaging of atmospheric state and flow variables, J. Atmos. Sci., 69, 1750-1757, 2012. 
Kowalski, A. S. and Argüeso, D.: Scalar arguments of the mathematical functions defining molecular and turbulent transport of heat and mass in compressible fluids, Tellus B, 63, 1059-1066, 2011.

Kowalski, A. S. and Serrano-Ortiz, P.: On the relationship between the eddy covariance, the turbulent flux, and surface exchange for a trace gas such as $\mathrm{CO}_{2}$, Bound.-Lay. Meteorol., 124, 129-141, 2007.

Kreith, F., Boehm, R. F., Raithby, G. D., Hollands, K. G. T., Suryanarayana, N. V., Carey, V. P., Chen, J. C., Lior, N., Shah, R. K., Bell, K. J., Moffat, R. J., Mills, A. F., Bergles, A. E., Swanson, L. W., Antonetti, V. W., Irvine, T. F., and Capobianchi, M.: Heat and Mass transfer, in: Mechanical Engineering Handbook, edited by: Kreith, F., CRC Press LLC, Boca Raton, 4-1-4-287, 1999.

Lee, X.: On micrometeorological observations of surface-air exchange over tall vegetation, Agr. Forest Meteorol., 91, 39-49, 1998.

Leuning, R.: Transport of gases into leaves, Plant Cell Environ., 6, 181-194, https://doi.org/10.1111/1365-3040.ep11587617, 1983.

Lienhard IV, J. H. and Lienhard V, J. H.: A heat transfer textbook, J. H. Lienhard V, Cambridge, MA, USA, 2000.

Mott, K. A. and Parkhurst, D. F.: Stomatal responses to humidity in air and helox, Plant Cell Environ., 14, 509-515, 1991.

Obukhov, A. M.: Turbulence in an atmosphere with a non-uniform temperature, Bound.-Lay. Meteorol., 2, 7-29, 1971.
Parkinson, K. J. and Penman, H. L.: A possible source of error in the estimation of stomatal resistance, J. Exp. Bot., 21, 405-409, 1970.

Stull, R. B. (Ed.): An introduction to boundary layer meteorology, in: Atmospheric and Oceanographic Sciences Library, Kluwer Academic Publishers, The Netherlands, 13, 670 pp., https://doi.org/10.1007/978-94-009-3027-8, 1988.

Suni, T., Rinne, J., Reissell, A., Altimir, N., Keronen, P., Rannik, Ü., Dal Maso, M., Kulmala, M., and Vesala, T.: Long-term measurements of surface fluxes above a Scots pine forest in Hyytiälä, southern Finland, 1996-2001, Boreal Environ. Res., 8, 287-301, 2003.

von Caemmerer, S. and Farquhar, G. D.: Some relationships between the biochemistry of photosynthesis and the gas exchange of leaves, Planta, 153, 376-387, https://doi.org/10.1007/bf00384257, 1981.

Wallace, J. M. and Hobbs, P. V.: Atmospheric science: an introductory survey, International Geophysics, edited by: Dmowska, R., Hartmann, D., and Rossby, H. T., Academic Press, Amsterdam, 483 pp., 2006.

Webb, E. K., Pearman, G. I., and Leuning, R.: Correction of flux measurements for density effects due to heat and water vapour transfer, Q. J. Roy. Meteor. Soc., 106, 85-100, 1980.

Wilczak, J. M., Oncley, S. P., and Stage, S. A.: Sonic anemometer tilt correction algorithms, Bound.-Lay. Meteorol., 99, 127-150, 2001. 